\title{
Tooth preparation for porcelain veneers - which technique do I use?
}

\author{
A study into the variations in the labial reduction of teeth prepared to receive porcelain veneers - \\ a comparison of three clinical techniques

\section{G. P. Cherukara, K. G. Seymour, D. Y. D. Samarawickrama and L. Zou Br Dent J 2002; 192: 401-404}

\section{Objectives}

Various techniques have been suggested to enable the operator to produce an even reduction of $0.5 \mathrm{~mm}$ of labial tooth enamel during preparation for a porcelain veneer. For example, in addition to the traditional free hand method, longitudinal or horizontal depth orientation grooves and the use of small round burs to produce dimples as depth guides have been suggested. However, there is no published data that compares how effective these techniques are at producing the 'ideal' veneer preparation. In this study three techniques were compared using the technique of co-ordinate metrology.

\section{Method}

A single operator using the above three techniques prepared 84 extracted teeth. Impressions of the prepared and unprepared teeth were scanned using a co-ordinate measuring machine (CMM). Measurements of maximum labial reduction along the mid-labial plane were taken and analysed.

\section{Results}

The study showed that among the three techniques studied the use of small round burs (D001-012), when used side on at an angle of $45^{\circ}$ to the tooth surface to produce dimples as depth guides, resulted in the greatest frequency of tooth reductions closer to the 'ideal' depth chosen for this study, ie within the $0.4 \mathrm{~mm}-0.6 \mathrm{~mm}$ range.

\section{Conclusion}

The study concluded that even after using techniques designed to produce consistent preparations, a single operator still produced preparations with considerable variation from the ideal. The study showed that among the three techniques compared the use of small round burs, when used side on at an angle of $45^{\circ}$ to the tooth surface to produce dimples as depth guides, resulted in the greatest frequency of tooth reduction closer to the 'ideal' depth chosen for this study only, ie within the $0.4 \mathrm{~mm}-0.6 \mathrm{~mm}$ range. It is stressed that this range may not be the ideal in all clinical situations.

\section{IN BRIEF}

- The study looked at three different techniques to achieve a depth range of 0.4-0.6 mm consistently for veneer tooth reduction.

- The use of a small round bur to produce dimples as depth guides was found to be superior to two other tooth reduction techniques investigated; viz depth orientation grooves and free hand reduction.

- The results also showed inconsistent preparation depth even when depth gauging techniques, investigated in this study, were used. This was because either some of the depth guides themselves or the subsequent reduction of the tooth surface was inaccurate.

- Therefore, even when such depth gauging techniques are used, the clinician should employ great caution while finishing the tooth preparation. This is because, it is possible to over prepare while finishing the preparation even if the depth guides were accurate.

\section{COMMENT}

Labial porcelain veneers provide good aesthetic results for a variety of situations such as staining, discolouration, surface defects, misshapen teeth etc. They are conservative restorations, and treatment with veneers involves minimal tooth reduction. However, it is preferable that during preparation only the enamel is involved since extension through to dentine may lead to sensitivity, need for temporary coverage, and the requirement of dentine bonding.

The usual reduction of $0.5 \mathrm{~mm}$ following the curvature of the labial surface can be difficult to achieve, and dentine is frequently exposed, particularly in the cervical region. A number of methods have been advocated for helping to ensure equal enamel reduction while following the labial contour, although no comparative data regarding effectiveness has been published.

In this study, one operator carried out all the preparations on 84 extracted human upper central incisors, 28 following each of three recommended methods of tooth reduction. Impressions were taken of the labial surfaces of the teeth before and after reduction and scanned by a co-ordinate measuring machine. The maximum reduction along the mid-labial plane was then calculated and recorded.

The results showed that, of the three techniques used, the one using a small round bur side on at an angle of $45^{\circ}$ gave an acceptable result in the range $0.4-0.6 \mathrm{~mm}$ more frequently than the other two methods. However, it may have been helpful if the authors had mentioned the time span under which the preparations were carried out, and the sequence of techniques. For example, were all the preparations carried out on the same occasion one after the other? Were all the preparations using each technique done together, or were they alternated? Nevertheless, the study does give help in deciding which technique to use to help in preparing teeth for labial porcelain veneers of the correct depth.

Ray B Winstanley, Reader in Restorative Dentistry Department of Adult Dental Health, Sheffield 